\title{
Control Cleansing
}

National Cancer Institute

\section{Source}

National Cancer Institute. Control Cleansing. NCI Thesaurus. Code C111376.

The process of washing the skin using a cleaning agent that functions as a standard of comparison in a control experiment. 